\title{
Editorial
}

\section{DEPARTAMENTO DE CAPACITACIÓN Y ENSEÑANZA EN ENFERMERÍA Y PERSONAL PARAMÉDICO}

Bajo la premisa de la misión del Instituto Nacional Neurología y Neurocirugía Manuel Velasco Suarez, que es desarrollar investigación científica, formar y capacitar recursos humanos calificados y otorgar servicios de atención médica de alta especialidad; el Departamento de Capacitación y Enseñanza en Enfermería y Personal Paramédico tiene como tarea primordial dar cumplimiento a esto, es por ello que cumple un papel de suma importancia en la capacitación y educación continua, que surge de las necesidades hospitalarias de atención, procedimental e innovación del cuidado que brindan los profesionales de enfermería y otras áreas paramédicas.

Es aquí donde se elaboran, diseñan y organizan actividades académicas que deberán apoyar estas demandas, dicha logística es plasmada en un Programa anual de capacitación para enfermería y personal paramédico el cual es publicado dentro del catálogo de actividades académicas del Instituto; asimismo este departamento trabaja de la mano con el Departamento de Desarrollo Profesional que depende directamente de la Subdirección de Enfermería, con quien se asumen responsabilidades tales como el Programa de capacitación, así como con las actividades propias que genera el Programa de Servicio Social y por supuesto con la apertura de campos clínicos como escenarios de práctica y estancias académicas de enfermería.

Para hacer posible lo anterior se establecen convenios de colaboración con los organismos educativos públicos y privados más importantes del país como, por ejemplo, la Universidad Nacional Autónoma de México, el Instituto Politécnico Nacional, la Universidad Autónoma Metropolitana, Benemérita Universidad de Puebla, Universidad de Morelos, Universidad de San Luis Potosí, Instituto Chiapas, Universidad Panamericana, Universidad. Modelo, Grupo Cedva, entre otros.
Estos convenios nos han llevado a poder compartir experiencias exitosas en la atención de los usuarios con daño neurológico lo que nos convierte en líderes en la atención de los padecimientos relacionados con el sistema nervioso.

Durante todo el año se realizan, aproximadamente, 45 cursos de capacitación y actualización dentro de las vertientes asistenciales, gerenciales de educación y formación, además, de aquellos establecidos como indispensables para las áreas de alta complejidad como son: RCP Básico y Avanzado, Inducciones al Puesto y, por supuesto, los encaminados a brindar Educación para la Salud dirigida a familiares y pacientes. Se tiene una cobertura aproximada de 1200 platicas anuales, las cuales, son impartidas con la colaboración de los pasantes en enfermería, además de cumplir con las asistenciales y de investigación, estas resultan ser también de gran importancias ya que, con materiales alusivos y atractivos a los temas, favorecen y atraen el interés del participante.

Otra actividad relevante es que el Instituto es SEDE de dos importantes Posgrados en Enfermería: Enfermería Neurológica y Adulto en Estado Crítico, dirigidos a enfermeras ya tituladas de nivel licenciatura y con mínimo 3 años de experiencia laborar, si bien estos Posgrados son de la Escuela Nacional de Enfermería y Obstetricia de la UNAM (ENEO-UNAM) el departamento de Capacitación y Enseñanza en Enfermería y Personal Paramédico es el responsable directo de su organización, dirección y desarrollo.

Mtra. María Verónica Balcázar Martínez Jefe del Depto. de Capacitación y Enseñanza en Enfermería Enf Neurol Vol. 16. No. 2 mayo - agosto 2017 